\title{
CPT AND STRINGS*
}

\author{
V. Alan KOSTELECKÝ and Robertus POTTING \\ Physics Department, Indiana University, Bloomington, IN 47405, USA
}

Received 21 March 1991

\begin{abstract}
We define the string space-time symmetries $C, P, T$ and their products. The effects of nonlocal interactions and the infinite number of particle fields are incorporated using string field theory. At the dynamical level, $C, P, T$ and their products are preserved by the open bosonic string. Only the product $C P T$ is dynamically preserved by the superstring, although a modified action can be constructed to preserve $C$. We show that these discrete symmetries may be spontaneously broken in a natural way by string interactions. In a favorable scenario, this might lead to $C P T$ violation in the $\mathrm{K}-\overline{\mathrm{K}}$ system just below the limits of present experiments.
\end{abstract}

\section{Introduction}

The $C P T$ theorem is a profound and general result for local relativistic quantum field theories in flat space-time. Originally proved by direct construction [1-5], it has since been rigorously derived in the framework of axiomatic field theory $[6,7]$. The theorem is supported by the results of precision experiments. For instance, measurements in the $\mathrm{K}-\overline{\mathrm{K}}$ system [8] have found no evidence for $C P T$ violation to a level of five parts in $10^{18}$.

String theory [9] provides a promising approach to a complete and coherent description of the fundamental interactions, including gravity. Several qualitative differences distinguish string models from standard local field theories. These include the presence of an infinite number of particle fields and the nonlocal nature of strings. In this paper, we study the implications of these nonstandard stringy features for the discrete spacetime symmetries $C, P, T$, and products. Our

\footnotetext{
* Dedicated to the memory of John Stewart Bell.
} 
analysis is performed using string field theory [10a, b, 11-17], which provides a convenient covariant formulation incorporating the nonlocal interactions and allowing for many-body effects.

One possibility for investigating $C P T$ in string theory is an axiomatic approach. At present, this is impractical because a suitable extension of the Wightman axioms has not yet been developed. An alternative is to generalize Pauli's original proof of the CPT theorem. For a local field theory, this involves defining the discrete symmetries $C, P, T$ on anticommuting irreducible Lorentz spinors and then proving that the $C P T$ transformation of any irreducible tensor product is determined by the rank of the tensor. In particular, Lorentz scalars (such as the lagrangian) are shown to be invariant. The chain of argument involves several assumptions, including the locality of field operators, the correspondence between spin and statistics, and the reality of observables. For strings, however, a proof of this type requires more information on the general structure of covariant string field theory than currently is available.

Instead, we adopt a practical approach applicable to any given string theory. It is an extension of the method described for local field theories in ref. [18], providing a clear basis for the definition of the discrete symmetries $C, P, T$ in the string context. The procedure is as follows. Begin with the free string theory expanded in particle modes. Construct $C, P, T$ operations so that $C$ interchanges particles and antiparticles and $P, T$ reduce correctly to the classical notions in the correspondence limit. These operations must leave invariant the free action and must be consistent with the quantization procedure. Next, consider the interacting theory. If the interaction terms are invariant under $C, P, T$ when the interacting fields are replaced by free fields, then the usual perturbative construction generates amplitudes that are also invariant. Note that verifying this invariance for strings requires rechecking the consistency of the quantization procedure. The point is that the quantization of the interacting theory is different from that of the free theory because the nonlocal interactions involve higher derivatives. A separate proof that anomalies are absent must therefore be given.

For any particular field theory of particles or strings, there may be several possible choices for $C, P, T$ at the level of the free action. For example, the free action for a real Lorentz-singlet field is invariant whether the field transforms under $P$ as a scalar or a pseudoscalar. Interactions can constrain this freedom, but even in their presence the possible assignment of $C, P, T$ transformations need not be unique. Additional constraints may be imposed if experimental input is used. For example, if a massless vector in a given string theory is interpreted as the photon then its transformation properties under $C, P, T$ are fixed by the macroscopic properties of the associated electric currents, just as occurs in quantum electrodynamics. For convenience, our $C, P, T$ assignments are made assuming that string modes in the massless sector are identified with particle fields of the standard model. 
The above discussion shows that violation of $C, P, T$ or products can occur at two different levels in a theory. First, it may be that the transformation cannot be defined as an invariance of the free theory. For instance, there is no acceptable definition preserving $\boldsymbol{C}$ or $\boldsymbol{P}$ for the free theory of a massless chiral fermion (neutrino) in four dimensions. Second, the interactions may not be invariant even though transformations on the free fields are well defined (and even though several acceptable choices exist for the free case).

For a given interacting string theory viewed in terms of its particle-field modes, the statement of invariance under $C, P, T$ or any products involves the assignment to infinitely many fields of transformation properties that are well defined for the free case and that are consistent with infinitely many interaction terms. A priori, this is a formidable task. Fortunately, the formalism of string field theory permits a neat packaging of the infinite number of assignments in terms of an appropriate transformation on the string field. Furthermore, the invariance of the action can then be demonstrated directly. For this reason our analysis here is largely performed at the level of the string field.

Since experimentally we see particles, not strings, any observable consequences from $C, P, T$ and products must be at the level of the particle modes. Therefore, once the properties is the string field in a given theory have been established, it is of interest to examine the induced features at the level of particle modes. Note that it is important to investigate the transformations of the higher-mass fields, even though they may not be directly observable. This is because the four-dimensional low-energy effective theory is obtained by integrating over heavy states, so an understanding of their transformation properties is crucial to determining the behavior of the effective theory under the action of $C, P, T$ and products.

We begin the analysis in sect. 2 with the case of the open bosonic string. The $C, P$, and $T$ transformations are introduced at the level of the string field in subsect. 2.1. It is useful to have them expressed in an oscillator realization, which is presented in subsect. 2.2. The transformation of the action is investigated in subsect. 2.3. Invariance at the quantum level requires verifying the absence of anomalies in the quantization procedure. This is considered in subsect. 2.4. The analysis is more involved than for standard particle field theories because of the intricacy of the canonical structure for the theory. In subsect. 2.5 we discuss the transformation properties of the particle modes of the string field, and we present explicit results for the first three levels.

The analysis is extended to the open superstring in sect. 3. Subsect. 3.1 contains definitions of the action of $C, P, T$ on the superstring fields, including their oscillator representations. The transformation of a class of superstring actions is considered in subsect. 3.2. A modified superstring action that preserves $C$ invariance in ten dimensions is discussed.

In sects. 2 and 3 we assume that the boundary conditions are invariant under $C, P, T$, i.e. that the vacuum is invariant. Sect. 4 considers the possibility of 
spontaneous breaking of these symmetries, with focus on $C P T$. Sect. 5 provides a summary of the results and a discussion, incorporating the implications of our analysis for realistic strings with gravity.

Our notation largely follows that of ref. [19], except for the ghost-number assignments as noted in the text. The Minkowski-space metrics we use have positive signature.

\section{The open bosonic string}

\subsection{TRANSFORMATION OF THE STRING FIELD}

We begin by considering the covariant field theory for the open bosonic string [10a]. The relevant string field $\Phi\left(X^{\mu}(\sigma), b(\sigma), c(\sigma)\right)$ is a functional of the string coordinates and the conformal ghosts. In this subsection, we present the transformations $C, P, T$ via their direct action on $\Phi$.

First, consider the parity operation $P$. We define it to reverse the sign of the spacelike components of $X^{\mu}(\sigma)$ :

$$
P: \quad X^{\mu}(\sigma) \rightarrow-X_{\mu}(\sigma) \quad\left(\eta_{00}=-1\right),
$$

and to leave unaffected the ghosts $b, c$. The action on the string field is

$$
P: \quad \Phi\left(X^{\mu}(\sigma), b(\sigma), c(\sigma)\right) \rightarrow \Phi\left(-X_{\mu}(\sigma), b(\sigma), c(\sigma)\right) .
$$

Next, consider the time-reversal operation $T$. We take it to change the sign of the timelike component $X^{0}(\sigma)$ :

$$
T: \quad X^{\mu}(\sigma) \rightarrow X_{\mu}(\sigma) .
$$

Again, the $b$ and $c$ ghosts are left unaffected. The action on the string field is

$$
T: \quad \Phi\left(X^{\mu}(\sigma), b(\sigma), c(\sigma)\right) \rightarrow \Phi\left(X_{\mu}(\sigma), b(\sigma), c(\sigma)\right) .
$$

Finally, consider the change-conjugation operation $C$. Classically, we take its action on $\Phi$ simply as complex conjugation:

$$
C: \quad \Phi\left(X^{\mu}(\sigma), b(\sigma), c(\sigma)\right) \rightarrow \Phi\left(X^{\mu}(\sigma), b(\sigma), c(\sigma)\right)^{*} .
$$

Note that the condition on the string ficld rendering the action real is

$$
\Phi\left(X^{\mu}(\sigma), b(\sigma), c(\sigma)\right)=\Phi\left(X^{\mu}(\pi-\sigma), b(\pi-\sigma), c(\pi-\sigma)\right)^{*} .
$$


For string fields satisfying this condition, the action of $C$ merely reverses the string orientation.

\subsection{FOCK-SPACE REPRESENTATION}

In this subsection we construct realizations for $C, P, T$ in the Fock-space basis, using the oscillator formalism of refs. [20-22].

Introduce the eigenstate $\left|X^{\mu}(\sigma), b(\sigma), c(\sigma)\right\rangle$ of the string coordinate operators $\hat{X}^{\mu}, \hat{b}, \hat{c}$ by the definition

$$
\begin{gathered}
\hat{X}^{\mu}(\sigma)\left|X^{\mu}(\sigma), b(\sigma), c(\sigma)\right\rangle=X^{\mu}(\sigma)\left|X^{\mu}(\sigma), b(\sigma), c(\sigma)\right\rangle, \\
\hat{b}(\sigma)\left|X^{\mu}(\sigma), b(\sigma), c(\sigma)\right\rangle=b(\sigma)\left|X^{\mu}(\sigma), b(\sigma), c(\sigma)\right\rangle \\
\hat{c}(\sigma)\left|X^{\mu}(\sigma), b(\sigma), c(\sigma)\right\rangle=c(\sigma)\left|X^{\mu}(\sigma), b(\sigma), c(\sigma)\right\rangle .
\end{gathered}
$$

The operator Fourier components are

$$
\begin{aligned}
& \hat{X}(\sigma)=\hat{x}_{0}+i \sum_{n \neq 0} \frac{1}{n} \alpha_{n} \cos n \sigma \\
& \hat{b}(\sigma)=\sum_{n=-\infty}^{\infty} b_{n} \cos n \sigma, \quad \hat{c}(\sigma)=\sum_{n=-\infty}^{\infty} c_{n} \sin n \sigma .
\end{aligned}
$$

The oscillators satisfy the relations

$$
\begin{aligned}
{\left[\alpha_{n}, \alpha_{m}\right] } & =n \delta_{n+m}, \quad\left\{c_{n}, b_{m}\right\}=\delta_{n+m, 0}, \quad\left\{c_{n}, c_{m}\right\}=\left\{b_{n}, b_{m}\right\}=0, \\
\hat{x}_{0}^{\dagger} & =\hat{x}_{0}, \quad \alpha_{n}^{\dagger}=\alpha_{-n}, \quad c_{n}^{\dagger}=c_{-n}, \quad b_{n}^{\dagger}=b_{-n} .
\end{aligned}
$$

This means $X(\sigma)$ and $b(\sigma)$ are hermitian, while $c(\sigma)$ and the ghost number operator $N_{b c}=\frac{1}{2}\left(c_{0} b_{0}-b_{0} c_{0}\right)+\Sigma_{n>0}\left(c_{-n} b_{n}-b_{-n} c_{n}\right)$ are antihermitian. The vacua $\left| \pm \frac{1}{2}\right\rangle$ have ghost number $\pm \frac{1}{2}$ and satisfy

$$
\begin{aligned}
& \alpha_{n}\left| \pm \frac{1}{2}\right\rangle=c_{n}\left| \pm \frac{1}{2}\right\rangle=b_{n}\left| \pm \frac{1}{2}\right\rangle=0 \quad(n>0), \\
& c_{0}\left|+\frac{1}{2}\right\rangle=b_{0}\left|-\frac{1}{2}\right\rangle=0, \quad c_{0}\left|-\frac{1}{2}\right\rangle=\left|+\frac{1}{2}\right\rangle, \quad b_{0}\left|+\frac{1}{2}\right\rangle=\left|-\frac{1}{2}\right\rangle, \quad\left\langle+\frac{1}{2} \mid-\frac{1}{2}\right\rangle=1 .
\end{aligned}
$$

The string field may now be written as

$$
\Phi\left(X^{\mu}(\sigma), b(\sigma), c(\sigma)\right)=\left\langle\Phi \mid X^{\mu}(\sigma), b(\sigma), c(\sigma)\right\rangle .
$$

This formalism permits us to express $C, P$ and $T$ in terms of oscillators. 
The first step is to identify the action of the parity transformation (2) on the operator eigenstates. We set

$$
\left|X^{\mu}(\sigma), b(\sigma), c(\sigma)\right\rangle \rightarrow\left|-X_{\mu}(\sigma), b(\sigma), c(\sigma)\right\rangle,
$$

so that $P$ reverses the sign of the spacelike oscillators and leaves the others unchanged. Note that $P$ affects the sign of the zero mode as well as the oscillator modes. This induces nontrivial signs in the transformation of particle fields, as is discussed in subsect. 2.5. The transformation can be written in operator form using two sets of oscillators,

$$
\left|X^{\mu}(\sigma), b(\sigma), c(\sigma)\right\rangle_{1} \rightarrow_{2}\langle P\rangle_{1}\left|X^{\mu}(\sigma), b(\sigma), c(\sigma)\right\rangle_{2},
$$

where

$$
\begin{aligned}
{ }_{2}\langle P\rangle_{1}= & { }_{2}\left\langle\frac{1}{2}\left|\exp \left[\sum_{n>0}\left(-\frac{1}{n} \sum_{\mu} \alpha_{-n}^{\mu(1)} \alpha_{n}^{\mu(2)}+b_{-n}^{(1)} c_{n}^{(2)}+c_{-n}^{(1)} b_{n}^{(2)}\right)+c_{0}^{(1)} b_{0}^{(2)}\right]\right|-\frac{1}{2}\right\rangle_{1} \\
& \times \int \mathrm{d} x_{0}^{\mu(2)} \delta^{d}\left(x_{0}^{\mu(1)}+x_{0 \mu}^{(2)}\right)
\end{aligned}
$$

Note the skewed position of the Lorentz indices! One can check that

$$
\hat{X}^{\mu(1)}(\sigma)_{2}\langle P\rangle_{1}=-{ }_{2}\langle P\rangle_{1} \hat{X}_{\mu}^{(2)}(\sigma)
$$

and thus

$$
\hat{X}^{\mu(1)}(\sigma)_{2}\langle P\rangle_{1}\left|X^{\mu}(\sigma), b(\sigma), c(\sigma)\right\rangle_{2}=-X_{\mu}(\sigma)_{2}\langle P\rangle_{1}\left|X^{\mu}(\sigma), b(\sigma), c(\sigma)\right\rangle_{2},
$$

as desired, and that the eigenvalues of the ghost operators are unchanged.

Next, we obtain the action (2) of $P$ on $\Phi$ in operator form. We use eq. (11) to convert the action from the operator eigenket to the string bra. This gives

$$
P: \quad{ }_{1}\langle\Phi| \rightarrow_{2}\left\langle\left.\Phi\right|_{1}\langle P\rangle_{2},\right.
$$

where it is now understood that $\left|X^{\mu}(\sigma), b(\sigma), c(\sigma)\right\rangle$ is left unchanged.

A similar treatment provides the operator form of the time-reversal transformation (4). The action on the coordinate-operator eigenket is to reverse the sign of the timelike oscillators:

$$
\left|X^{\mu}(\sigma), b(\sigma), c(\sigma)\right\rangle \rightarrow\left|X_{\mu}(\sigma), b(\sigma), c(\sigma)\right\rangle .
$$


In operator form this becomes

$$
\left|X^{\mu}(\sigma), b(\sigma), c(\sigma)\right\rangle_{1} \rightarrow_{2}\langle T\rangle_{1}\left|X^{\mu}(\sigma), b(\sigma), c(\sigma)\right\rangle_{2},
$$

where

$$
\begin{aligned}
{ }_{2}\langle T\rangle_{1}= & { }_{2}\left\langle\frac{1}{2}\left|\exp \left[\sum_{n>0}\left(\sum_{\mu} \frac{1}{n} \alpha_{-n}^{\mu(1)} \alpha_{n}^{\mu(2)}+b_{-n}^{(1)} c_{n}^{(2)}+c_{-n}^{(1)} b_{n}^{(2)}\right)+c_{0}^{(1)} b_{0}^{(2)}\right]\right|-\frac{1}{2}\right\rangle_{1} \\
& \times \int \mathrm{d} x_{0}^{\mu(2)} \delta^{d}\left(x_{0}^{\mu(1)}-x_{0 \mu}^{(2)}\right) .
\end{aligned}
$$

As desired, this satisfies

$$
\hat{X}^{\mu(1)}(\sigma)_{2}\langle T\rangle_{1}={ }_{2}\langle T\rangle_{1} \hat{X}_{\mu}^{(2)}(\sigma)
$$

and hence

$$
\hat{X}^{\mu(1)}(\sigma)_{2}\langle T\rangle_{1}\left|X^{\mu}(\sigma), b(\sigma), c(\sigma)\right\rangle_{2}=X_{\mu}(\sigma)_{2}\langle T\rangle_{1}\left|X^{\mu}(\sigma), b(\sigma), c(\sigma)\right\rangle_{2},
$$

while the eigenvalues of the ghost operators are unchanged. Using eq. (11), we can again transfer the action to $\langle\Phi|$ :

$$
{ }_{1}\langle\Phi| \rightarrow_{2}\left\langle\left.\Phi\right|_{1}\langle T\rangle_{2}\right.
$$

A different approach is needed to cast charge conjugation (5) in operator form. From eq. (11) we have

$$
C: \quad\left\langle\Phi \mid X^{\mu}(\sigma), b(\sigma), c(\sigma)\right\rangle \rightarrow\left\langle X^{\mu}(\sigma), b(\sigma), c(\sigma) \mid \Phi\right\rangle,
$$

where we define $\left\langle X^{\mu}(\sigma), b(\sigma), c(\sigma)\right| \equiv\left(\left|X^{\mu}(\sigma), b(\sigma), c(\sigma)\right\rangle\right)^{\dagger}$. To interpret this as an action on $\langle\Phi|$ only, we introduce a "conjugation vertex" ${ }_{21}\langle C|$ satisfying

$$
{ }_{2}\left\langle X^{\mu}(\sigma), b(\sigma), c(\sigma)\right|={ }_{21}\left\langle C \mid X^{\mu}(\sigma), b(\sigma), c(\sigma)\right\rangle_{1} .
$$

Then, it follows that

$$
C: \quad{ }_{2}\langle\Phi| \rightarrow_{21}\langle C \mid \Phi\rangle_{1} \text {. }
$$


The conjugation vertex is symmetric and is explicitly given by

$$
|C\rangle_{12}=\exp \left[-b_{0}^{(1)} c_{0}^{(2)}+\sum_{n>0}\left(-\frac{1}{n} \alpha_{-n}^{(1)} \cdot \alpha_{-n}^{(2)}-b_{-n}^{(1)} c_{-n}^{(2)}-b_{-n}^{(2)} c_{-n}^{(1)}\right)\right]\left|+\frac{1}{2}\right\rangle_{1}\left|-\frac{1}{2}\right\rangle_{2} .
$$

It is interesting to compare the conjugation vertex $|C\rangle_{12}$ to the usual two-vertex $\left|V_{2}\right\rangle_{12}$, given by

$$
\left|V_{2}\right\rangle_{12}=\exp \left[-b_{0}^{(1)} c_{0}^{(2)}+\sum_{n>0}(-1)^{n}\left(-\frac{1}{n} \alpha_{-n}^{(1)} \cdot \alpha_{-n}^{(2)}-b_{-n}^{(1)} c_{-n}^{(2)}-b_{-n}^{(2)} c_{-n}^{(1)}\right)\right]\left|+\frac{1}{2}\right\rangle_{1}\left|-\frac{1}{2}\right\rangle_{2}
$$

The two differ in their overlap equations. The standard two-vertex $\left\langle V_{2}\right|$ satisfies

$$
\begin{gathered}
\left(X^{(1)}(\sigma)-X^{(2)}(\pi-\sigma)\right)\left|V_{2}\right\rangle_{12}=0, \\
\left(b^{(1)}(\sigma)-b^{(2)}(\pi-\sigma)\right)\left|V_{2}\right\rangle_{12}=0, \\
\left(c^{(1)}(\sigma)+c^{(2)}(\pi-\sigma)\right)\left|V_{2}\right\rangle_{12}=0,
\end{gathered}
$$

while for the conjugation vertex ${ }_{21}\langle C|$ we demand that

$$
\begin{gathered}
\left(X^{(1)}(\sigma)-X^{(2)}(\sigma)\right)|C\rangle_{12}=0, \\
\left(b^{(1)}(\sigma)-b^{(2)}(\sigma)\right)|C\rangle_{12}=0, \\
\left(c^{(1)}(\sigma)-c^{(2)}(\sigma)\right)|C\rangle_{12}=0 .
\end{gathered}
$$

The choice of $\langle C|$ in eq. (26) (instead of an analogous equation using $\left\langle V_{2}\right|$ ) is dictated by the requirements that the interaction term be invariant (see subsect. 2.3) and that the massless vector transform in the usual way (see subsect. 2.5).

\subsection{TRANSFORMATION OF THE ACTION}

With the operator definitions of $C, P, T$, we can directly check the transformation properties of the action for the open bosonic string,

$$
\begin{aligned}
S & =\frac{1}{2 \alpha^{\prime}} \int \Phi * Q \Phi+\frac{g}{3} \int \Phi * \Phi * \Phi \\
& \equiv \frac{1}{2 \alpha^{\prime}}{ }_{21}\left\langle V_{2}|| \Phi\right\rangle_{1} Q|\Phi\rangle_{2}+\frac{g}{3}{ }_{321}\left\langle V_{3}|| \Phi\right\rangle_{1}|\Phi\rangle_{2}|\Phi\rangle_{3} .
\end{aligned}
$$


First, apply the charge-conjugation operation to the action $S$. The effect of this can be seen as follows. From the overlap conditions on $|C\rangle$ and on the vertices, one can show that the conjugation vertex is BRST invariant,

$$
\left(Q^{(1)}+Q^{(2)}\right)|C\rangle_{12}=0
$$

Furthermore, applying the conjugation vertex to the legs of $\left\langle V_{2}\right|$ or $\left\langle V_{3}\right|$ gives rise to the hermitian conjugate $\left|V_{2}\right\rangle$ or $\left|V_{3}\right\rangle$. Using also that $\left\langle V_{2}\right|$ is Grassmann odd, these properties lead to the identities

$$
\begin{aligned}
{ }_{43}\left\langle V_{2}\left|Q^{(4)}\right| C\right\rangle_{13}|C\rangle_{24} & =Q^{(2)}\left|V_{2}\right\rangle_{12}, \\
{ }_{654}\left\langle V_{3}|| C\right\rangle_{14}|C\rangle_{25}|C\rangle_{36} & =\left|V_{3}\right\rangle_{123} .
\end{aligned}
$$

From these equations and using $Q^{\dagger}=Q$, we see that the charge-conjugation operation $C$ maps $S$ to its hermitian conjugate. Imposing the usual reality condition on the fields, the action becomes hermitian and hence also invariant under $C$.

To examine the effect of $\boldsymbol{P}$ on the action, take the transformed action and apply the operation (14) to the vertices. This reverses the sign of spacelike $\alpha_{n}^{\mu}$ oscillators, including the zero modes. The net effect is to leave the vertices invariant, as can be seen in two ways. The first involves verifying the invariance of the overlap equations. The second is to analyse the explicit form of the vertices. The point here is that $\left|V_{2}\right\rangle$ and $\left|V_{3}\right\rangle$ are exponentials of expressions quadratic in oscillators, so the minus signs cancel pairwise.

This second argument shows that $P$ invariance of the action follows from the connection between the spacetime physics and the worldsheet physics. The vertices must have a $P$-invariant form because they can be viewed as path integrals of a free (and hence quadratic) world-sheet action on a particular world-sheet configuration coupled to external-string momentum distributions. The gaussian integrals yield Neumann functions, which are quadratic exponentials in the external string oscillators. Note in particular that one must transform all string fields simultaneously for invariance because the vertices contain terms mixing oscillators from different strings.

For the $T$ operation a similar argument can be made, involving sign changes for timelike oscillators. One additional complication arises from the antiunitary nature of $T$, which means all constants in the action are complex conjugated. For the kernels ${ }_{21}\left\langle V_{2}\right| Q^{(2)}$ and ${ }_{321}\left\langle V_{3}\right|$, this is equivalent to hermitian conjugation followed 
by transposition using the inverted conjugation vertex $\langle C|$ :

$$
\begin{aligned}
{ }_{21}\left\langle V_{2}\right| Q^{(2)} & \rightarrow{ }_{42}\left\langle\left. C\right|_{31}\left\langle C\left|Q^{(4)}\right| V_{2}\right\rangle_{34},\right. \\
{ }_{321}\left\langle V_{3}\right| & \rightarrow{ }_{63}\left\langleC | _ { 5 2 } \left\langle\left. C\right|_{41}\left\langle C|| V_{3}\right\rangle_{456} .\right.\right.
\end{aligned}
$$

These, however, are identities following from eq. (33).

We conclude that for fields satisfying the reality condition (6), the action (31) is invariant under each of the symmetries $C, P$, and $T$. Invariance under products follows; in particular, the action for the open bosonic string is invariant under $C P T$ despite the nonlocal nature of strings.

\subsection{QUANTIZATION}

Invariance of the classical action does not necessarily ensure the correct transformation of quantum expectation values. We need to argue $C P T$ invariance of the quantization procedure too. Furthermore, the gauge symmetry of the string field action (31) implies the need for consistent CPT transformation properties for ghosts.

Canonical quantization of the theory (31) is intricate because the cubic interaction introduces arbitrary time derivatives, which complicates the structure of the conjugate momenta. Since the theory has gauge invariance, there will certainly be first-class constraints. Moreover, these form an infinitely reducible tower (gauge invariance of the gauge invariance of...), which induces a nested structure of ghosts (ghosts for ghosts for...) [23,24]. There are probably also second-class constraints [25], which have to be included in the measure or eliminated by the introduction of Dirac brackets [26]. Although a rigorous quantization along these lines has not been performed, we can nevertheless show by general arguments that any such quantization must preserve $C P T$.

Consider a generic field component $f$. The conjugate momentum is

$$
\pi\left(x_{0}\right)=\frac{\delta S}{\delta \partial_{0} f\left(x_{0}\right)}
$$

where the time derivative is with respect to the time component of the center-ofmass coordinate $x_{0}^{0}$. If $f$ transforms under $C P T$ as

$$
f\left(x_{0}\right) \rightarrow \epsilon f^{\dagger}\left(-x_{0}\right) \quad(\epsilon= \pm 1),
$$

then it follows from (35) and hermiticity of $S$ that

$$
\pi\left(x_{0}\right) \rightarrow-\epsilon \pi^{\dagger}\left(-x_{0}\right)
$$


For real fields, the hermitian conjugation plays no role. Thus, each conjugate momentum transforms with an extra sign relative to the corresponding field. This result holds also for conjugate momenta associated with higher time derivatives of the fields.

In the canonical treatment, eq. (35) is used to express $\partial_{0} f$ in terms of $f$ and $\pi$. From the transformations of the fields and momenta, the hamiltonian

$$
H(\pi, f)=-L+\int \mathrm{d}^{d-1} x \pi \partial_{0} f
$$

is invariant under CPT (for real fields). Defining the fundamental Poisson brackets by

$$
\left\{\pi\left(x_{0}\right), f\left(y_{0}\right)\right\}=\delta^{d-1}\left(x_{0}-y_{0}\right),
$$

it follows that for the $C P T$-transformed field we have

$$
\left\{\pi^{C P T}\left(x_{0}\right), f^{C P T}\left(y_{0}\right)\right\}=-\delta^{d-1}\left(x_{0}-y_{0}\right),
$$

due to the extra sign in the transformation of the momentum. At the quantum level, this sign is consistent with the complex conjugation of $i \hbar$ generated by the antilinearity of $C P T$.

Primary constraints on the phase-space variables arise whenever it is not possible to invert eq. (35) for all degrees of freedom. From the transformations defined above, we see that these constraints transform homogeneously, i.e. they acquire at most an overall sign under $C P T$. Requiring the primary constraints to be preserved in time yields secondary constraints, given by the Poisson brackets of the constraints with the hamiltonian. Since the hamiltonian is CPT invariant, the secondary constraints also transform homogeneously. The same holds true for tertiary and higher-order constraints.

The constraints can be split, following Dirac, into first class (those that have weakly vanishing Poisson bracket with all constraints) and second class (the complement). The first-class constraints generate gauge invariances. Quantization introduces Lagrange multipliers and ghosts [27]. The CPT transformations of the latter are determined by the values of $\epsilon$, and they ensure the effective action for the extended phase space is CPT invariant. When the theory is reducible, the procedure is repeated for the extra variables. The second-class constraints can be either included in the measure or converted to first-class constraints via Dirac brackets. In either case, the resulting expressions transform correctly under $C P T$.

\subsection{TRANSFORMATION OF THE COMPONENT FIELDS}

The expansion (8) of the string coordinates in Fourier modes permits the string field to be expressed in particle fields. In this subsection, we obtain the particle-field 
$C, P, T$ properties that are induced by the corresponding transformations on the string field.

Particle fields in the string-field expansion are functions of the center-of-mass coordinate $x_{0}^{\mu}$. In terms of real component fields, we write [19]

$$
\begin{aligned}
|\Phi\rangle=\left\langle\phi\left(x_{0}\right)\right. & +A_{\mu}\left(x_{0}\right) \alpha_{-1}^{\mu}+i \alpha\left(x_{0}\right) b_{-1} c_{0}+\frac{1}{\sqrt{2}} i B_{\mu}\left(x_{0}\right) \alpha_{-2}^{\mu}+\frac{1}{\sqrt{2}} B_{\mu \nu}\left(x_{0}\right) \alpha_{-1}^{\mu} \alpha_{-1}^{\nu} \\
& \left.+\beta_{0}\left(x_{0}\right) b_{-2} c_{0}+\beta_{1}\left(x_{0}\right) b_{-1} c_{-1}+i k_{\mu}\left(x_{0}\right) b_{-1} c_{0} \alpha_{-1}^{\mu}+\ldots\right)\left|-\frac{1}{2}\right\rangle
\end{aligned}
$$

The transformations of the particle fields are found by applying to $|\Phi\rangle$ the explicit operators $|C\rangle_{12},{ }_{1}\langle P\rangle_{2},{ }_{1}\langle T\rangle_{2}$, and comparing the result component-by-component with $|\Phi\rangle$.

Particular care must be taken when considering $T$ or any product containing it. These antiunitary transformations complex-conjugate all coefficients of the particle fields, including the modes of $X(\sigma), b(\sigma), c(\sigma)$. The result may be an additional sign for the $T$ transformations of certain particle fields. This sign can be found by explicit calculation, but a quick method is to use the bracket formulation (11). Substituting 1 in $\langle\Phi|$ for the particle field in question and zero for all others, the multiplying expression is real if ${ }_{2}\langle\Phi|={ }_{21}\langle C \mid \Phi\rangle_{1}$, imaginary if ${ }_{2}\langle\Phi|=-{ }_{21}\langle C \mid \Phi\rangle_{1}$.

For some of the low-level particle fields the transformations thus obtained are summarized in table 1 . The CPT transformations given coincide with those in standard particle field theory. In this basis, CPT invariance of the action can be directly inferred using Lorentz invariance and hermiticity. This follows because the overall sign from CPT corresponds to a sign for each Lorentz index and because

TABLE 1

List of $C, P, T$ and $C P T$ iransfurmations for particle fields in the expansion (41) of the open-bosonic-string field. The meaning of the tilde is defined by $\tilde{O}^{\mu} \equiv O_{\mu}$

\begin{tabular}{ccccc}
\hline Field & $C$ & $P$ & $T$ & $C P T$ \\
\hline$\phi\left(x_{0}\right)$ & $\phi^{\dagger}\left(x_{0}\right)$ & $\phi\left(-\tilde{x}_{0}\right)$ & $\phi\left(\tilde{x}_{0}\right)$ & $\phi^{\dagger}\left(-x_{0}\right)$ \\
$A_{\mu}\left(x_{0}\right)$ & $-A_{\mu}^{\dagger}\left(x_{0}\right)$ & $-A^{\mu}\left(-\tilde{x}_{0}\right)$ & $-A^{\mu}\left(\tilde{x}_{0}\right)$ & $-A_{\mu}^{\dagger}\left(-x_{0}\right)$ \\
$\alpha\left(x_{0}\right)$ & $-\alpha^{\dagger}\left(x_{0}\right)$ & $\alpha\left(-\tilde{x}_{0}\right)$ & $-\alpha\left(\tilde{x}_{0}\right)$ & $\alpha^{\dagger}\left(-x_{0}\right)$ \\
$B_{\mu}\left(x_{0}\right)$ & $B_{\mu}^{\dagger}\left(x_{0}\right)$ & $-B^{\mu}\left(-\tilde{x}_{0}\right)$ & $B^{\mu}\left(\tilde{x}_{0}\right)$ & $-B_{\mu}^{\dagger}\left(-x_{0}\right)$ \\
$B_{\mu \nu}\left(x_{0}\right)$ & $B_{\mu \nu}^{\dagger}\left(x_{0}\right)$ & $B^{\mu \nu}\left(-\tilde{x}_{0}\right)$ & $B^{\mu \nu}\left(\tilde{x}_{0}\right)$ & $B_{\mu \nu}^{+}\left(-x_{0}\right)$ \\
$\beta_{0}\left(x_{0}\right)$ & $\beta_{0}^{\dagger}\left(x_{0}\right)$ & $\beta_{0}\left(-\tilde{x}_{0}\right)$ & $\beta_{0}\left(\tilde{x}_{0}\right)$ & $\beta_{0}^{\dagger}\left(-x_{0}\right)$ \\
$\beta_{1}\left(x_{0}\right)$ & $\beta_{1}^{\dagger}\left(x_{0}\right)$ & $\beta_{1}\left(-\tilde{x}_{0}\right)$ & $\beta_{1}\left(\tilde{x}_{0}\right)$ & $\beta_{1}^{\dagger}\left(-x_{0}\right)$ \\
$k_{\mu}\left(x_{0}\right)$ & $k_{\mu}^{\dagger}\left(x_{0}\right)$ & $-k^{\mu}\left(-\tilde{x}_{0}\right)$ & $k^{\mu}\left(\tilde{x}_{0}\right)$ & $-k_{\mu}^{\dagger}\left(-x_{0}\right)$ \\
\hline
\end{tabular}


the vertices are Lorentz-invariant expressions, for which all Lorentz indices are contracted pairwise.

\section{The open superstring}

\subsection{TRANSFORMATION OF THE SUPERSTRING FIELDS}

A covariant field theory for the superstring [10b,11-13] involves two superstring fields, one $(A)$ for the Neveu-Schwarz sector and one $(\Psi)$ for the Ramond sector. In this subsection the transformation of these fields under $C, P$, and $T$ is established by extending the arguments presented in subsects. 2.1 and 2.2 for the open bosonic string.

For the superstring the coordinates $X^{\mu}(\sigma), c(\sigma), b(\sigma)$ have as supersymmetric partners the spinor $\psi^{\mu}(\sigma)$ and the commuting superghosts $\gamma(\sigma)$ and $\beta(\sigma)$. Their mode expansions incorporating both coordinates and momenta are

$$
\begin{gathered}
\psi^{\mu}(\sigma)=\sum_{n=-\infty}^{\infty} d_{n}^{\mu} \mathrm{e}^{i n \sigma}, \quad \gamma(\sigma)=\sum_{n=-\infty}^{\infty} \gamma_{n} \mathrm{e}^{i n \sigma}, \quad \beta(\sigma)=\sum_{n=-\infty}^{\infty} \beta_{n} \mathrm{e}^{i n \sigma}, \\
\left\{d_{m}^{\mu}, d_{n}^{\nu}\right\}=\eta^{\mu \nu} \delta_{m+n, 0}, \quad\left[\gamma_{m}, \beta_{n}\right]=\delta_{m+n, 0} \\
\left(d_{n}^{\mu}\right)^{\dagger}=2 d_{0}^{0} d_{-n}^{\mu} d_{0}^{0}, \quad\left(\gamma_{n}\right)^{\dagger}=\gamma_{-n}, \quad\left(\beta_{n}\right)^{\dagger}=-\beta_{-n}
\end{gathered}
$$

where $n$ and $m$ take half-integral values in the Neveu-Schwarz sector and integral values in the Ramond sector.

Introducing the antihermitian ghost-number operators $N_{\beta \gamma}^{\mathrm{NS}}=-\Sigma_{r>0}\left(\gamma_{-r} \boldsymbol{\beta}_{r}+\right.$ $\left.\beta_{-r} \gamma_{r}\right)$ and $N_{\beta \gamma}^{\mathrm{R}}=-\frac{1}{2}\left(\gamma_{0} \beta_{0}+\beta_{0} \gamma_{0}\right)-\sum_{n>0}\left(\gamma_{-n} \beta_{n}+\beta_{-n} \gamma_{n}\right)$, we label the superghost vacua by their ghost number. They satisfy

$$
\begin{array}{lll}
d_{r}^{\mu}|N\rangle=\gamma_{r+N}|N\rangle=\beta_{r-N}|N\rangle=0, & r \geqslant \frac{1}{2}, & N= \pm 1 \quad \text { (NS), } \\
d_{n}^{\mu}|N\rangle=\gamma_{n+N-\frac{1}{2}}|N\rangle=\beta_{n-N-\frac{1}{2}}|N\rangle=0, & n \geqslant 1, & N= \pm \frac{1}{2} \quad \text { (R). }
\end{array}
$$

In the Ramond sector, the zero modes $d_{0}^{\mu}$ of $\psi^{\mu}$ appear in a special way. Their vacuum is degenerate and can be labeled by a spinor index. The $d_{0}^{\mu}$ then act as gamma matrices on the kets $|\alpha\rangle$ and $|\bar{\alpha}\rangle$ according to

$$
d_{0}^{\mu}|\alpha\rangle=\sqrt{\frac{1}{2}}|\bar{\beta}\rangle \Gamma_{\bar{\beta} \alpha}^{\mu}, \quad d_{0}^{\mu}|\bar{\alpha}\rangle=\sqrt{\frac{1}{2}}|\beta\rangle \Gamma_{\beta \bar{\alpha}}^{\mu},
$$

in the notation of ref. [28]. The barred and unbarred indices refer to opposite chiralities. The (normalized) kets have opposite Grassmann parity. In what follows, we denote the Fock-space expansions of $A$ and $\Psi$ by $|A\rangle$ and $|\Psi\rangle$. 
For the parity operation we need to generalize the $\langle P\rangle$ operator in eq. (14). In analogy with the bosonic case and to preserve world-sheet supersymmetry, we take the parity operation to reverse the spacelike coordinates of both $X^{\mu}$ and $\psi^{\mu}$, while leaving the ghosts and superghosts unaffected. Thus, we define

$$
P: \quad\left\{\begin{array}{l}
|A\rangle_{1} \rightarrow_{2}\langle P\rangle_{1}|A\rangle_{2} \\
|\Psi\rangle_{1} \rightarrow_{2}\langle P\rangle_{1}|\Psi\rangle_{2}
\end{array}\right.
$$

with

$$
{ }_{2}\langle P\rangle_{1} \equiv \begin{cases}{ }_{2}\left\langle P^{B}\right\rangle_{1}{ }_{2}\left\langle P^{\mathrm{NS}}\right\rangle_{1} & \text { (Neveu-Schwarz) } \\ { }_{2}\left\langle P^{B}\right\rangle_{1}{ }_{2}\left\langle P^{\mathrm{R}}\right\rangle_{1} & \text { (Ramond), }\end{cases}
$$

where ${ }_{2}\left\langle P^{B}\right\rangle_{1}$ applies the parity operation to the $X, b$ and $c$ coordinates as in eq. (14). For the supersymmetric partners we impose the overlap equations

$$
\begin{gathered}
\psi^{\mu(1)}(\sigma)_{2}\langle P\rangle_{1}=-{ }_{2}\langle P\rangle_{1} \psi_{\mu}^{(2)}(\sigma), \\
\gamma^{(1)}(\sigma)_{2}\langle P\rangle_{1}={ }_{2}\langle P\rangle_{1} \gamma^{(2)}(\sigma), \\
\beta^{(1)}(\sigma)_{2}\langle P\rangle_{1}={ }_{2}\langle P\rangle_{1} \beta^{(2)}(\sigma) .
\end{gathered}
$$

The oscillator realizations of these conditions are

$$
\begin{aligned}
& { }_{2}\left\langle P^{\mathrm{NS}}\right\rangle_{1}={ }_{2}\left\langle-1\left|\exp \left[\sum_{r \geqslant \frac{1}{2}} \sum_{\mu}-d_{-r}^{(1) \mu} d_{r}^{(2) \mu}+\sum_{r \geqslant \frac{3}{2}} \beta_{-r}^{(1)} \gamma_{r}^{(2)}-\sum_{r \geqslant-\frac{1}{2}} \gamma_{-r}^{(1)} \beta_{r}^{(2)}\right]\right| 1\right\rangle_{1}, \\
& { }_{2}\left\langle P^{\mathrm{R}}\right\rangle_{1}={ }_{2}\left\langle-\frac{1}{2}\left|\sqrt{2} d_{0}^{0(1)} \exp \left[\sum_{n \geqslant 1} \sum_{\mu} d_{-n}^{(1) \mu} d_{n}^{(2) \mu}+\sum_{n \geqslant 1} \beta_{-n}^{(1)} \gamma_{n}^{(2)}-\sum_{n \geqslant 0} \gamma_{-n}^{(1)} \beta_{n}^{(2)}\right]\right| \frac{1}{2}\right\rangle_{1} .
\end{aligned}
$$

In eq. (49) it is implicitly understood that a trace is taken over a set of normalized vacuum basis states. The correct overlap equations for the $d_{0}$ then follow from $d_{0}^{\mu} d_{0}^{0}=-d_{0}^{0} d_{0 \mu}$. Note that because $d_{0}^{0}$ also anticommutes with the $d_{n}^{\mu}$ oscillators, $n \neq 0$, there is an additional sign associated with the quadratic $d_{n}^{\mu}$ term in the exponent. This sign is understood to be included, as are similar signs associated with the $b$ and $c$ ghosts.

The time-reversal operation can be defined by generalizing the operator ${ }_{1}\langle T\rangle_{2}$ of eq. (20). Define

$$
T: \quad\left\{\begin{array}{l}
|A\rangle_{1} \rightarrow_{2}\langle T\rangle_{1}|A\rangle_{2} \\
|\Psi\rangle_{1} \rightarrow_{2}\langle T\rangle_{1}|\Psi\rangle_{2}
\end{array}\right.
$$


The overlap equations with the coordinate superpartners are

$$
\begin{gathered}
\psi^{\mu(1)}(\sigma)_{2}\langle T\rangle_{1}={ }_{2}\langle T\rangle_{1} \psi_{\mu}^{(2)}(\sigma), \\
\gamma^{(1)}(\sigma)_{2}\langle T\rangle_{1}={ }_{2}\langle T\rangle_{1} \gamma^{(2)}(\sigma), \\
\beta^{(1)}(\sigma)_{2}\langle T\rangle_{1}={ }_{2}\langle T\rangle_{1} \beta^{(2)}(\sigma) .
\end{gathered}
$$

These equations have the oscillator realizations

$$
\begin{aligned}
& { }_{2}\left\langle T^{\mathrm{NS}}\right\rangle_{1}={ }_{2}\left\langle-1\left|\exp \left[\sum_{r \geqslant \frac{1}{2}} \sum_{\mu} d_{-r}^{(1) \mu} d_{r}^{(2) \mu}+\sum_{r \geqslant \frac{3}{2}} \beta_{-r}^{(1)} \gamma_{r}^{(2)}-\sum_{r \geqslant-\frac{1}{2}} \gamma_{-r}^{(1)} \beta_{r}^{(2)}\right]\right| 1\right\rangle_{1}, \\
& { }_{2}\left\langle T^{\mathrm{R}}\right\rangle_{1}={ }_{2}\left\langle-\frac{1}{2}\left|\Gamma^{11} \sqrt{2} d_{0}^{0(1)} \exp \left[\sum_{n \geqslant 1} \sum_{\mu}-d_{-n}^{(1) \mu} d_{n}^{(2) \mu}+\sum_{n \geqslant 1} \beta_{-n}^{(1)} \gamma_{n}^{(2)}-\sum_{n \geqslant 0} \gamma_{-n}^{(1)} \beta_{n}^{(2)}\right]\right| \frac{1}{2}\right\rangle_{1} .
\end{aligned}
$$

Here, $\Gamma^{11}$ is the normalized product of all the $d_{0}$. It anticommutes with $d_{0}^{\mu}$.

The action of charge conjugation on the superstring field is more subtle. Recall that for the open bosonic string we defined it by complex conjugation of the string field, eq. (24). Via the coordinate eigenstate (7), this transformation could be rewritten as a transformation on $\langle\Phi|$ using the conjugation vertex $|C\rangle$, eq. (26). This procedure does not extend naturally to the superstring because the coordinate superpartner $\psi^{\mu}$ cannot be separated from its conjugate momentum, so there is no natural choice of coordinate eigenstate. Indeed, $\psi^{\mu}$ is its own canonical conjugate.

Fortunately, there is a natural way to generalize the conjugation vertex $|C\rangle$. The idea is to define a set of overlap equations that unambiguously determine $|C\rangle$. An acceptable and natural generalization of eq. (30) to an arbitrary field $f(\sigma)$ of conformal dimension $J$ is

$$
\left(f^{(1)}(\sigma)-(-1)^{J} f^{(2)}(-\sigma)\right)|C\rangle_{12}=0 .
$$

An advantage of this overlap is that it has an explicit oscillator realization. Its simplicity also eases our analysis in subsect. 3.2 of the transformation of the action.

The formula (54) is motivated by interpreting the conjugation vertex as arising from operator expectation values, just as for the interaction vertices [20-22, 29, 30]. The goal here is to overlap $f^{(1)}(\sigma)$ with $f^{(2)}(\sigma)$. However, any two-vertex implicitly reverses the direction of $\tau$ because it converts a bra to a ket. To maintain conformal invariance on the complex strip $\xi=\tau+i \sigma$, one must also reverse the direction of $\sigma$. These reversals generate the conformal factor $\left(\mathrm{d} \xi^{\prime} / \mathrm{d} \xi\right)^{J}=(-1)^{J}$. 
Note that in the variable $z=\exp (\xi)$ the effect is to mix the upper and lower halves of the complex $z$ plane, i.e. string 1 overlaps with the double of string 2 .

Eq. (54) suggests that the superstring conjugation vertex should satisfy

$$
\begin{gathered}
\left(\psi^{\mu(1)}(\sigma)-i \psi^{\mu(2)}(-\sigma)\right)|C\rangle_{12}=0, \\
\left(\gamma^{(1)}(\sigma)+i \gamma^{(2)}(-\sigma)\right)|C\rangle_{12}=0, \\
\left(\beta^{(1)}(\sigma)+i \beta^{(2)}(-\sigma)\right)|C\rangle_{12}=0,
\end{gathered}
$$

since the coordinate superpartners $\psi^{\mu}, \gamma, \beta$ have conformal dimensions $\frac{1}{2},-\frac{1}{2}, \frac{3}{2}$, respectively. We have chosen a sign for the square root in the second term of each overlap; the physics is independent of this choice.

The explicit realization of the superstring conjugation vertex for the NeveuSchwarz sector is

$$
\left|C^{\mathrm{NS}}\right\rangle_{12}=\exp \left[i \sum_{r \geqslant \frac{1}{2}} d_{-r}^{(1)} \cdot d_{-r}^{(2)}-i \sum_{r \geqslant \frac{3}{2}} \beta_{-r}^{(1)} \gamma_{-r}^{(2)}+i \sum_{r \geqslant-\frac{1}{2}} \beta_{-r}^{(2)} \gamma_{-r}^{(1)}\right]|1\rangle_{1}|-1\rangle_{2}
$$

For the Ramond sector we find

$$
\left|C^{\mathrm{R}}\right\rangle_{12}=\exp \left[i \sum_{n \geqslant 1} d_{-n}^{(1)} \cdot d_{-n}^{(2)}-i \sum_{n \geqslant 0} \beta_{-n}^{(1)} \gamma_{-n}^{(2)}+i \sum_{n \geqslant 1} \beta_{-n}^{(2)} \gamma_{-n}^{(1)}\right]\left|\frac{1}{2}\right\rangle_{1}\left|-\frac{1}{2}\right\rangle_{2}|0\rangle_{12}
$$

Note that the overlap conditions (55) are insensitive to an arbitrary phase, which can be incorporated in the realization (56), (57). This phase can be chosen so that the massless fermion component field $\psi\left(x_{0}\right)$ in the expansion of the string field $\Psi$ transforms without a phase, i.e. $C: \psi \rightarrow C \bar{\psi}^{\mathrm{T}}$. Note also that these vertices $|C\rangle$ are not symmetric, unlike the conjugation vertex for the bosonic string. For the Ramond conjugation vertex, the vacuum $|0\rangle_{12}$ of the zero modes of $\psi^{\mu}$ is annihilated by $d_{0}^{\mu(1)}-i d_{0}^{\mu(2)}$. This is the vacuum used in the construction of the Ramond two-vertex. Explicitly, choosing the Majorana-Weyl representation and using the spinor notation of [28], we have

$$
|0\rangle_{12}=\sum_{\alpha \bar{\beta}} C_{\alpha \bar{\beta}}|\alpha\rangle_{1}|\bar{\beta}\rangle_{2}+i \sum_{\bar{\alpha} \beta} C_{\bar{\alpha} \beta}|\bar{\alpha}\rangle_{1}|\beta\rangle_{2}
$$

Here, $C_{\alpha \bar{\beta}}$ and $C_{\bar{\alpha} \beta}$ are components of a charge-conjugation matrix with $C=\Gamma^{0}$. 
We can thus define the action of charge conjugation $C$ on the superstring fields by

$$
C: \quad\left\{\begin{array}{l}
|A\rangle_{1} \rightarrow_{2}\left\langle A \mid C^{\mathrm{NS}}\right\rangle_{12} \\
|\Psi\rangle_{1} \rightarrow_{2}\left\langle\bar{\Psi} \mid C^{\mathrm{R}}\right\rangle_{12} .
\end{array}\right.
$$

Here, $\langle\bar{\Psi}|$ involves multiplication with $\Gamma^{0} \equiv \sqrt{2} d_{0}^{0}$, as is customary in taking the conjugate spinor. This factor arises from the hermiticity properties of the $d_{0}^{\mu}$ and is necessary for a $C$-invariant action.

\subsection{TRANSFORMATION OF THE ACTION}

Several actions have been proposed for the open superstring [10b,11-13]. We use the general formulation of ref. [12], for which the action is

$$
S=\frac{1}{2 \alpha^{\prime}} \int Y_{-2} A * Q A+\frac{g}{3} \int Y_{-2} A * A * A+\frac{1}{\sqrt{\alpha^{\prime}}} \int Y \Psi * Q \Psi+2 g \sqrt{\alpha^{\prime}} \int Y A * \Psi * \Psi .
$$

Here, $Y$ and $Y_{-2}$ are (inverse) picture-changing operators [31], the latter carrying ghost number -2 . The action $S$ differs from the original proposal [10b] in that the string fields are based in the 0-picture rather than the (-1)-picture, which is the origin of the factors of $Y_{-2}$. This formulation avoids the divergences in the original proposal caused by the collision of picture-changing operators $X$ at the vertex midpoint [32]. Note that there are various possiblities for the insertions of $Y$ and $Y_{-2}$. One can take $Y$ either at $\sigma=\pi / 2$ or at $-\pi / 2$. Moreover, for $Y_{-2}$ one can choose either a symmetric, nonchiral combination of insertions or one of 15 possible chiral insertions at $\sigma= \pm \pi / 2$.

In this subsection we investigate the effects of the transformations $C, P, T$ and products on the action (60). The analysis is an extension of that for the open bosonic string in subsect. 2.3.

Arguments similar to those for the open bosonic string show that the vertices, the BRST operator, and the picture-changing operators are invariant under the action of both $\langle P\rangle$ and $\langle T\rangle$. However, one additional subtlety appears: neither $P$ nor $T$ leaves invariant the covariant GSO projection operator [33,34] applied on the Ramond field and required for supersymmetry:

$$
\mathscr{P}_{\mathrm{R}}=\frac{1}{2}\left(1+\Gamma_{11}(-)^{N_{\mathrm{R}}}\right), \quad N_{\mathrm{R}}=\sum_{n>0}\left(d_{-n} \cdot d_{n}+\beta_{-n} \gamma_{n}-\gamma_{-n} \beta_{n}\right)+\beta_{0} \gamma_{0} .
$$

This is because chirality in ten dimensions is inverted by $P$ and $T$ (cf. the four-dimensional case, for which chirality is changed by $C$ and $P$ ). 
Both charge conjugation and time reversal involve application of the conjugation operators $\left|C^{\mathrm{NS}}\right\rangle$ and $\Gamma_{0}\left|C^{\mathrm{R}}\right\rangle$ (we include explicitly the $\Gamma_{0}$ from the Ramond field in (59)). The issue in this case is whether application of the appropriate conjugation vertices gives rise to the hermitian conjugate of the action.

First, analyse the situation ignoring the midpoint insertions in the vertices. Using the overlap equations yields

$$
\left(Q^{(1)}+Q^{(2)}\right)|C\rangle_{12}=0 \quad(\text { NS and R })
$$

Inspection of the overlap equations and use of the identity $Q^{\mathrm{NS} \dagger}=Q^{\mathrm{NS}}$ shows that the two- and three-vertices involving only $A$ transform as needed, i.e. their overlap equations transform into those of the hermitian-conjugate vertices.

A similar analysis for the vertices $\left|V_{\mathrm{RR}}^{2}\right\rangle$ and $\left|V_{\mathrm{RRNS}}^{3}\right\rangle$ involving the Ramond string field (using the identity $d_{0}^{0} Q^{\mathrm{R}}=-Q^{\mathrm{R} \dagger} d_{0}^{0}$ ) can also be performed. For this case, two additional subtleties arise. First, a constraint appears on the reality condition for the Ramond-sector field. The point is that a priori this condition contains an arbitrary phase. However, requiring invariance of the action fixes this phase. Second, a difficulty arises because the Ramond oscillators obey symmetric (rather than antisymmetric) boundary conditions. For instance, one overlap condition is $[22,28]$

$$
\left(\psi^{\mu(1)}( \pm \sigma)+i \psi^{\mu(2)}( \pm(\pi-\sigma))\left|V_{\mathrm{RR}}^{2}\right\rangle=0\right.
$$

Conjugating this vertex with the above prescription generates overlap conditions differing by a sign from those of the hermitian-conjugate vertex. The same happens for the superghost overlap equations. Thus, for general fields the action is not invariant.

However, the presence of the GSO projection avoids this problem. Expanding the Ramond string field about the unbarred vacua, thereby explicitly exhibiting the contribution of the $d_{0}^{\mu}$ oscillators to the GSO projection, one sees that only even products of $\psi^{\mu}, \beta$ and $\gamma$ appear. This means, for example, that the missing minus sign in eq. (63) can be absorbed by redefining the oscillators in one of the string fields by a sign. Thus, in effect the two-vertex is invariant. A similar argument can be made for $\left|V_{\mathrm{RRNS}}^{3}\right\rangle$, where again the minus signs can be absorbed in one of the Ramond fields.

We are left with the issue of the transformation of picture-changing operators under application of $|C\rangle$. As discussed above, the operators $Y, Y_{-2}$ are inserted at $\sigma=\pi / 2$ and $/$ or $-\pi / 2$. However, $|C\rangle$ involves an inversion of $\sigma$, i.e. any insertion at $\pi / 2$ is converted to $-\pi / 2$ and vice versa. Chiral terms are not invariant under this interchange. Therefore, the presence of a chiral term in the action implies the violation of $C$ and $T$. 
This violation may be seen explicitly at the level of the particle theory by expanding the string fields in the Fock-space basis. For example, the free Ramond-sector lagrangian expanded to the first massive level [35] displays several off-diagonal terms connecting the massless and the massive states. Mixing terms of this type between odd and even mass levels arise directly from the insertion of $Y$, and they break $C$ invariance.

The difficulty can be fixed by modifying the definition of the action (60). To avoid the $C$-violating terms in the vertices with Ramond string fields, we must insert a nonchiral combination $\frac{1}{2}(Y(i)+Y(-i))$. Requiring the absence of anomalies from the collision of picture-changing operators then forces the choice $Y_{-2}=$ $Y(i) Y(-i)$.

We therefore consider instead the action

$$
\begin{aligned}
S^{\prime}= & \frac{1}{2 \alpha^{\prime}} \int Y(i) Y(-i) A * Q A+\frac{g}{3} \int Y(i) Y(-i) A * A * A \\
& +\frac{1}{\sqrt{\alpha^{\prime}}} \int \frac{1}{2}(Y(i)+Y(-i)) \Psi * Q \Psi+2 g \sqrt{\alpha^{\prime}} \int \frac{1}{2}(Y(i)+Y(-i)) A * \Psi * \Psi .
\end{aligned}
$$

This action is preserved by the (modified) gauge transformations

$$
\begin{aligned}
& \delta A=\frac{1}{\sqrt{2 \alpha^{\prime}}} Q \Lambda+\sqrt{\frac{\alpha^{\prime}}{2}} g(A * \Lambda-\Lambda * A)+\frac{1}{2 \sqrt{2}} \alpha^{\prime} g(X(i)+X(-i))(\Psi * \chi-\chi * \Psi), \\
& \delta \Psi=\frac{1}{\sqrt{2 \alpha^{\prime}}} Q \chi+\sqrt{\frac{\alpha^{\prime}}{2}} g(A * \chi-\chi * A)+\sqrt{\frac{\alpha^{\prime}}{2}} g(\Psi * \Lambda-\Lambda * \Psi) .
\end{aligned}
$$

The troublesome terms appearing in $S$ are now absent from $S^{\prime}$, as may be seen explicitly by expanding the string fields in the Fock-space basis. No odd-even level-mixing terms appear. The action $S^{\prime}$ is $C$ invariant.

In summary, the class of superstring actions $(60)$ violates parity and time-reversal invariance due to its chiral nature (GSO projection), although the product $C P T$ is preserved. Furthermore, each term with a chiral picture-changing insertion ( $Y$ and possibly $Y_{-2}$ ) violates both charge-conjugation and time-reversal invariance, calthough again $C P T$ is preserved. The modified action (64) also violates parity and time-reversal invariance, as is to be expected from the chiral nature of the theory. However, it preserves $C$. Both actions are invariant under the product $C P T$, despite the nonlocality of the superstring. 


\section{Spontaneous symmetry breaking}

In the previous sections we have considered exclusively dynamical $C, P, T$ or product symmetries. However, as usual, the solutions to the equations of motion are invariant only if in addition the boundary conditions are symmetric. In the present context, this means that the vacuum must also be invariant under $C, P, T$ or products. Nonzero expectation values for some fields can spoil these invariances. Although it is possible to break spontaneously the individual symmetries $C, P, T$ in string theory, this is not of primary interest because they can readily be broken at the dynamical level. Explicit examples are provided by the superstring, as we have seen in sect. 3. Of more interest is the possibility of spontaneous breaking of the product $C P T$, which is dynamically preserved in the cases considered here. In fact, in string theory there exists a natural mechanism for the violation of $C P T$ that does not occur in ordinary particle theories, as we now describe.

It is known that string interactions incorporate couplings among particle states that are absent in standard particle gauge theories [36]. In the framework of string field theory, these couplings appear as interaction terms in the action that violate naive gauge invariance but that are in fact compatible with the full string gauge invariance [19]. Among the implications of such terms is the possibility of spontaneous breaking of the higher-dimensional Lorentz symmetry [36]. An example in the open bosonic string is provided by the tachyon-vector-vector coupling $\phi A_{\mu} A^{\mu}$. If the tachyon expectation value is nonzero [37] and of the appropriate sign, the vector $A_{\mu}$ acquires a negative squared mass. The resulting expectation value $\left\langle A_{\mu}\right\rangle$ breaks the 26-dimensional Lorentz symmetry.

If this mechanism occurs it can also break $C P T$ invariance. Consider a field component $f$ that changes sign under $C P T$. When $f$ acquires a nonzero expectation value and is shifted, any interaction terms involving $f$ generate a $C P T$-violating piece. If, as usual, scalars do not change sign under $C P T, f$ must have nontrivial Lorentz structure. Therefore, the CPT breaking is necessarily associated with breaking of the higher-dimensional Lorentz symmetry. The mechanism there : avoids no-go results on spontaneous $C P T$ breaking [38].

The question naturally arises as to whether spontaneous $C P T$ breaking in the underlying higher-dimensional theory could lead to any observable effects in four dimensions. This is a model-dependent issue. Four-dimensional CPT breaking is indeed a possibility, however, because the $d$-dimensional transformation $C P T_{d}$ reduces in four dimensions to the product of $C P T_{4}$ with a discrete symmetry. This may be seen as follows. For a generic $d$-dimensional field $f(x, y)$ depending on four dimensions $x$ and $d-4$ dimensions $y$, the mode expansion can be viewed as

$$
f(x, y)=\sum_{k, m} f_{k m}(x) X_{k m}(y),
$$


where the sums are over the four-dimensional Lorentz representation content (labeled by $k$ ) of the $d$-dimensional field $f$ and over the modes (labeled by $m$ ) of each representation. The action of the $d$-dimensional transformations on $f$ is

$$
\begin{aligned}
C_{d}: & f(x, y) \rightarrow c_{d} f^{*}(x, y), \\
P_{d}: & f(x, y) \rightarrow p_{d} f\left(x_{0},-\vec{x},-y\right), \\
T_{d}: & f(x, y) \rightarrow t_{d} f\left(-x_{0}, \vec{x}, y\right), \\
C P T_{d}: & f(x, y) \rightarrow c p t_{d} f^{*}(-x,-y),
\end{aligned}
$$

where $c_{d}, p_{d}$ and $t_{d}$ are constants or matrices appropriately acting on $f$ according to its $d$-dimensional Lorentz properties. Similarly, the four-dimensional transformations on the component fields $f_{k m}$ are

$$
\begin{aligned}
C_{4}: & f_{k m}(x) \rightarrow c_{4} f_{k m}^{*}(x), \\
P_{4}: & f_{k m}(x) \rightarrow p_{4} f_{k m}\left(x_{0},-\vec{x}\right), \\
T_{4}: & f_{k m}(x) \rightarrow t_{4} f_{k m}\left(-x_{0}, \vec{x}\right), \\
C P T_{4}: & f_{k m}(x) \rightarrow c p t_{4} f_{k m}^{*}(-x) .
\end{aligned}
$$

From these equations and the mode expansion (66) we see that $C P T_{d}$ reduces in the compactified theory to $C P T_{4} \times G$, where $G$ is a discrete group (possibly containing only the identity). Thus, since $C P T_{d}$ does contain $C P T_{4}$, spontaneous breaking of $C P T_{d}$ could lead to spontaneous breaking of $C P T_{4}$.

If this mechanism does lead to any four-dimensional CPT breaking, it is accompanied by four-dimensional Lorentz breaking. Since no experimental evidence of either is apparent, any such string effects must be suppressed in a realistic low-energy effective theory. This could occur, for example, if the terms inducing $C P T$ violation in the shifted theory involved only higher modes. The induced violation in the effective field theory would then be suppressed by the usual decoupling theorem [39].

Several experiments test $C P T$ invariance purely in particle physics, and there is even one proposal [40] for testing $C P T$ invariance using the gravitational field. At present, the most sensitive test for $C P T$ violation (and Lorentz violation) is the measurement of the $\mathrm{K}^{\mathbf{0}}-\overline{\mathrm{K}}^{\mathbf{0}}$ mass difference. Using experimental information from $K$ decays and imposing unitarity via the Bell-Steinberger relation [41] permits 
bounds to be placed on the ratio $\left|\left(M_{\mathrm{K}}-M_{\overline{\mathrm{K}}}\right) / M_{\mathrm{K}}\right|$. For a clear account of some of the issues involved in the analysis see, for example, ref. [42].

As we have discussed above, in an optimistic scenario stringy effects could manifest themselves as $C P T$ breaking, suppressed by some ratio of a light scale to the Planck scale. Suppose we are given a realistic effective four-dimensional model based on a fundamental string theory, containing spontaneous $C P T$ violation without zeroth-order effects. The order of magnitude at which $C P T$ violation might appear in the $\mathrm{K}-\overline{\mathrm{K}}$ system can then be crudely estimated. The relevant light scale presumably lies in the region between $M_{\mathrm{K}} \approx 0.5 \mathrm{GeV}$ and $M_{\mathrm{W}} \approx 100 \mathrm{GeV}$, so one might anticipate stringy $C P T$-violating effects in the range $M_{\mathrm{K}} / M_{\mathrm{P}} \approx 5 \times 10^{-20}$ to $M_{\mathrm{W}} / M_{\mathrm{P}} \approx 10^{-17}$. This may be compared to the current experimental bound [8], $\left|\left(M_{\mathrm{K}}-M_{\overline{\mathrm{K}}}\right) / M_{\mathrm{K}}\right|<5 \times 10^{-18}$. From this we see that $C P T$ violation might occur at limits just beyond present experiments.

\section{Discussion}

Strings are qualitatively different from point particles because they are extended objects. This suggests the existence of qualitatively new physical effects in theories based on strings. However, direct measurements at the Planck scale are far beyond current experiments. The standard folklore dismisses the possibility that any such effects are observable at our present low energies. This is based on the viewpoint that at low energy any underlying string theory can be described by an effective field theory based on point particles, which purportedly cannot directly display features of extended objects.

This folklore may be too pessimistic. It overlooks the possibility that the low-energy effective theory could have an unusual appearance when viewed purely as a particle theory. Our point is that uniquely stringy effects, i.e. ones that do not occur in normal point-particle physics, by definition must violate some principle accepted as valid for standard particle theories. Remnants of such effects could therefore appear in a low-energy model in the form of terms not normally found in particle theory.

One way to proceed is to identify some stringy effect occurring at the Planck scale and then to consider its manifestation in a low-energy effective theory. The extended nature of strings can suggest candidate stringy effects. For example, string gauge invariance is different from particle gauge invariance. The two agree only to low order in the gauge coupling. As a consequence, the low-energy effective action can contain terms that violate naive particle gauge invariance [36]. These terms can lead to observable effects, including photon and graviton masses [43].

The possibility we have considered in this paper is that the nonlocal nature of strings might lead to a violation of $C P T$ invariance in the low-energy effective theory. This symmetry is particularly well suited for such a study because it has 
already been experimentally tested to a high degree of precision. Moreover, it is the only experimentally identified discrete symmetry that is not known to be violated at some level.

In the preceding sections we have studied the $C P T$ properties of the action for the open bosonic string and for the open superstring. Our analysis uses string field theory, which is a covariant many-body formalism that incorporates nonlocal effects. At the present level of development, it is impractical to provide a general proof of $C P T$ invariance either by group-theoretic arguments [1-5] or by axiomatic methods [6,7]. Instead, our analysis is on a case-by-case basis, constructing acceptable $C, P, T$ transformations of the free string fields and examining properties of the interactions.

Our arguments demonstrate that the action of the open bosonic string is invariant under the individual transformations $C, P, T$ as well as the product $C P T$. In contrast, the action of the open superstring preserves only the product $C P T$. The violations of $P$ and $T$ are to be expected in a chiral ten-dimensional theory. However, $C$ nonconservation is a consequence of the choice of $C$-breaking insertions of picture-changing operators. This feature is evaded by a modified form of the superstring action along with a change in the associated string gauge invariance. The resulting action preserves $C$ and $C P T$ while breaking $P$ and $T$, as desired.

We have shown that the possibility does exist that $C P T$ is spontaneously broken in a natural and stringy way. If this breaking includes four-dimensional $C P T$, it could appear at the level of the low-energy effective string theory. In the $\mathbf{K}-\overline{\mathrm{K}}$ system, this might yield CPT violation of order one part in $10^{19}$. This level of precision might be attained in experiments in the near future.

It is interesting that the differences between particle and string field theories are insufficient to cause dynamical $C P T$ violation. It appears that the nonlocalities are too smooth to induce CPT breakdown. This situation can be contrasted with apparently simpler nonlocal particle-theory models, such as certain self-conjugate multiplets of fermionic fields [44], that are known to violate CPT. Also, the presence of an infinite number of particle fields has no effect because each field enters as a finite irreducible representation of the Lorentz group. There are no infinite irreducible representations, for which in particle field theory it is known that $C P T$ violation can occur [45].

The arguments we have given evidently incorporate some of the standard assumptions, such as the presence of a flat background, the completeness of the asymptotic Hilbert space, and the validity of the perturbation series. The analysis used both reality of observables (hermiticity of the action) and spin-statistics (appropriate quantum commutation relations and role [46] of the GSO projection). Note, however, that the usual requirement of normal-ordered interactions is unnecessary here. The cyclic symmetry of the string vertices ensures that the interaction terms are of the correct form. 
The space-time $C P T$ considered in this paper should be distinguished from world-sheet $C P T$ [47-49]. The latter has been used to show equality of spacetime masses [47]. Moreover, our results can extend to strings the standard arguments showing that the masses and lifetimes of particles and antiparticles are equal and that cross sections are unaffected by $C P T$ reversal $[3,5]$. These facts suggest world-sheet $C P T$ might be closely connected to space-time $C P T$. Note that using first-quantized string theory and in the context of a flat background disregarding nonlocalities, ref. [50] has argued space-time $C P T$ invariance of string scattering amplitudes.

An important issue is the incorporation of closed strings into the analysis. This is technically harder because the string field interactions are now nonpolynomial [14-17]. Moreover, the presence of gravity introduces some conceptual issues. Even in standard general relativity the situation is complicated [50-53]. The possibilities of curved space-time, negative (gravitational) energy density, asymptotic incompleteness (due to black-hole states), and nonlocality (from the quantized metric) are all specifically excluded in the usual proofs of the CPT theorem. It has even been suggested that gravity must violate $C P T$ to generate the observed time asymmetry of the universe [54]. The restriction to asymptotically flat spacetimes may permit a weaker notion of CPT invariance, but "strong" CPT is probably violated by the loss of quantum coherence at horizons. All these features are also present for closed strings.

In any case, curved backgrounds cannot presently be treated in the context of the covariant field theory for the closed bosonic string. An analysis might be possible in the framework of nonlinear sigma models, for example, but such on-shell methods would not incorporate the nonlocal features of interest here. We remark in passing that $C P T$ invariance of the nonpolynomial closed-string field theory probably does hold in a flat background and might be established by generalizations of the arguments of sect. 2 above. Also, couplings of the type that can lead to spontaneous CPT violation are known to be present [55].

In conclusion, the nonlocalities in string theory are too smooth to damage dynamical spacetime $C P T$ invariance. However, a natural and stringy mechanism for spontaneous $C P T$ breaking exists that might lead to effects just beyond present experimental limits.

This paper is dedicated to the memory of John Stewart Bell, a deep thinker who had a long-standing interest in the CPT theorem (stemming from his independent derivation of it in the early 1950s [3]) and with whom we enjoyed speculating about its validity in string theory.

We thank Abhay Ashtekar, Pete Carruthers, Terry Goldman, Don Page, and Stuart Samuel for stimulating discussions. This research was supported in part by the United States Department of Energy under contract number DE-AC0284ER40125. 


\section{References}

[1] J. Schwinger, Phys. Rev. 82 (1951) 914

[2] G. Lüders, Det. Kong. Danske Videnskabernes Selskab Mat.-fysiske Meddelelser 28, no. 5 (1954); Ann. Phys. (N.Y.) 2 (1957) 1

[3] J.S. Bell, Birmingham University thesis (1954); Proc. Roy. Soc. London A231 (1955) 479

[4] W. Pauli, in W. Pauli, ed., Niels Bohr and the development of physics (McGraw-Hill, New York, 1955) p. 30

[5] G. Lüders and B. Zumino, Phys. Rev. 106 (1957) 345

[6] R.F. Streater and A.S. Wightman, PCT, spin and statistics, and all that (Benjamin Cummings, Reading, 1964)

[7] R. Jost, The general theory of quantized fields (AMS, Providence, 1965)

[8] R. Carosi et al., Phys. Lett. B237 (1990) 303

[9] M.B. Green, J.H. Schwarz and E. Witten, Superstring Theory, vols. 1 and 2 (Cambridge Univ. Press, Cambridge, 1987)

[10a] E. Witten, Nucl. Phys. B268 (1986) 253

[10b] E. Witten, Nucl. Phys. B276 (1986) 291

[11] O. Lechtenfeld and S. Samuel, Phys. Lett. B213 (1988) 431

[12] C. Preitschopf, C.B. Thorn and S.A. Yost, Nucl. Phys. B337 (1990) 363

[13] I.Ya. Aref'eva, P. Medvedev and A. Zubarev, Nucl. Phys. B341 (1990) 464

[14] T. Kugo, H. Kunimoto and K. Suehiro, Phys. Lett. B226 (1989) 48

[15] T. Kugo and K. Suehiro, Nucl. Phys. B337 (1990) 434

[16] M. Kaku, Phys. Rev. D41 (1990) 3734

[17] M. Saadi and B. Zweibach, Ann. Phys. (N.Y.) 192 (1989) 213

[18] R.G. Sachs, Prog. Theor. Phys. Suppl. 86 (1986) 336

[19] V.A. Kostelecký and S. Samuel, Nucl. Phys. B336 (1990) 263; Phys. Rev. Lett. 64 (1990) 2238

[20] E. Cremmer, A. Schwimmer and C. Thorn, Phys. Lett. B179 (1986) 57

[21] S. Samuel, Phys. Lett. B181 (1986) 255

[22] D.J. Gross and A. Jevicki, Nucl. Phys. B283 (1987) 1, 225; B293 (1987) 29

[23] C. Thorn, Nucl. Phys. B287 (1987) 61

[24] M. Bochicchio, Phys. Lett. B188 (1987) 330; 193 (1987) 31

[25] R. Potting, C. Taylor and B. Velikson, Phys. Lett. B198 (1987) 184

[26] P.A.M. Dirac, Proc. Roy. Soc. London A246 (1958) 326

[27] E.S. Fradkin and G.A. Vilkovisky, Phys. Lett. B55 (1975) 224;

I.A. Batalin and G.A. Vilkovisky, Phys. Lett. B69 (1977) 309;

E.S. Fradkin and M.A. Vasiliev, Phys. Lett. B72 (1977) 70;

E.S. Fradkin and T.E. Fradkina, Phys. Lett. B72 (1978) 343

[28] K. Suehiro, Nucl. Phys. B296 (1988) 333; Prog. Theor. Phys. 78 (1987) 1151

[29] G.T. Horowitz and S.P. Martin, Nucl. Phys. B296 (1988) 220

[30] A. LeClair, M.E. Peskin and C.R. Preitschopf, Nucl. Phys. B317 (1989) 411, 464

[31] D. Friedan, E. Martinec and S. Shenker, Nucl. Phys. B271 (1986) 93

[32] C. Wendt, Nucl. Phys. B314 (1989) 209

[33] F. Gliozzi, J. Scherk and D. Olive, Nucl. Phys. B122 (1977) 253

[34] H. Terao and S. Uehara, Phys. Lett. B168 (1986) 70

[35] J.P. Yamron, Phys. Lett. B187 (1987) 67

[36] V.A. Kostelecký and S. Samuel, Phys. Rev. D39 (1989) 683; Phys. Rev. Lett. 63 (1989) 224; Phys. Rev. D40 (1989) 1886

[37] V.A. Kostelecký and S. Samuel, Phys. Lett. B207 (1988) 169

[38] C. Vafa and E. Witten, Phys. Rev. Lett. 53 (1984) 535; Nucl. Phys. B234 (1984) 173

[39] T. Appelquist and J. Carazzone, Phys. Rev. D11 (1975) 2856

[40] T. Goldman and M.M. Nieto, Phys. Lett. B112 (1982) 437

[41] J.S. Bell and J. Steinberger, in Proc. Oxford Int. Conf. on Elementary Particles 1965, ed. T.R. Walsh, A.E. Taylor, R.G. Moorhouse and B. Southworth (Rutherford Laboratory, Chilton, Didcot, 1966) p. 195 
[42] N.W. Tanner and R.H. Dalitz, Ann. Phys. (N.Y.) 171 (1986) 463

[43] V.A. Kostelecký and S. Samuel, Phys. Rev. Lett. 66 (1991) 1811

[44] P. Carruthers, Phys. Rev. Lett. 18 (1967) 353; Phys. Lett. B26 (1968) 158

[45] A.I. Oksak and I.T. Todorov, Commun. Math. Phys. 11 (1968) 125

[46] V.A. Kostelecký, O. Lechtenfeld, W. Lerche, S. Samuel and S. Watamura, Nucl. Phys. B288 (1987) 173

[47] E. Witten, Commun. Math. Phys. 109 (1987) 536

[48] G. Moore and N. Seiberg, Nucl. Phys. B313 (1989) 16

[49] H. Sonoda, Nucl. Phys. B326 (1989) 135

[50] S.W. Hawking, Commun. Math. Phys. 87 (1982) 395; Phys. Rev. D32 (1985) 2489

[51] D. Page, Phys. Rev. Lett. 44 (1980) 301; Gen. Rel. Grav. 14 (1982) 299; Phys. Rev. D32 (1985) 2496

[52] R.M. Wald, Phys. Rev. D21 (1980) 2742

[53] A. Ashtekar, email communication

[54] R. Penrose, in General relativity: An Einstein centenary survey, ed. S.W. Hawking and W. Israel (Cambridge Univ. Press, Cambridge, 1979)

[55] V.A. Kostelecký and S. Samuel, Phys. Rev. D42 (1990) 1289 